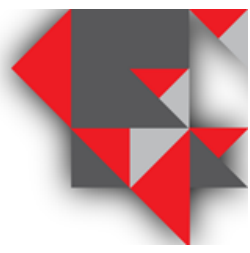

\title{
Condiciones de acceso a la tecnología para el trabajo remoto en México en tiempos de pandemia por COVID-19
}

\author{
Ingrid N. Pinto-López ${ }^{1}$, Cynthia M. Montaudon Tomas ${ }^{2}$ y Alicia L. Yáñez-Moneda ${ }^{3}$ \\ ${ }^{1}$ UPAEP Universidad, Facultad de Inteligencia de Negocios, Escuela de Negocios. Puebla, México. \\ Ingrid.pinto@upaep.mx, 21 Sur \#1103, Barrio de Santiago (+52) 2222299400 \\ ${ }^{2}$ UPAEP Universidad, Facultad de Administración de Empresas, Escuela de Negocios. Puebla, México. \\ Cynthiamaria.montaudon@upaep.mx, 21 Sur \#1103, Barrio de Santiago (+52) 2222299400 \\ ${ }^{3}$ UPAEP Universidad, Dirección de Hospitalidad y Turismo, Escuela de Negocios. Puebla, México. \\ alicialucreciayanez@upaep.mx,21 Sur\#1103,Barrio de Santiago (+52) 2222299400
}

Información del artículo revisado por pares

Fecha de aceptación: junio-2021

Fecha de publicación en línea: diciembre-2021

DOI: https://doi.org/10.29105/vtga7.2-55

\section{Resumen}

Abstract

Este artículo analiza las condiciones de acceso a la This article analyzes the conditions of access to technology tecnología para el trabajo remoto que los profesionales de for remote work that professionals from different economic los diferentes sectores económicos en México han vivido sectors in Mexico have experienced during the period of durante el período de confinamiento derivado de la lockdown derived from the health contingency caused by contingencia sanitaria por la COVID-19. El COVID-19. The data collection was carried out between levantamiento de datos se realizó entre cinco y siete five and seven months after the confinement measures meses después de que en México se decretaran las were decreed in Mexico and the professional activities medidas de confinamiento y se migraran las actividades were migrated to telework mode, giving the participants profesionales a modalidad teletrabajo dando tiempo a los time to adapt to the new normal. A scale was developed participantes para adaptarse a la nueva normalidad. Una and validated and subsequently applied to 1,685 escala fue desarrollada y validada y posteriormente participants in 30 of the 32 states of the Mexican Republic. aplicada a 1685 participantes en 30 de los 32 estados de The results show relevant data on indicators related to the la república mexicana. Los resultados muestran datos conditions of access to technology, training and tools for relevantes sobre indicadores relacionados con las the remote work.

condiciones de acceso a la tecnología, la capacitación y herramientas para el trabajo remoto.

Palabras clave: trabajo remoto, tecnología, confinamiento, sector económico, México, COVID-19.

Keywords: remote work, technology, lockdown, economic sector, Mexico, COVID-19

JEL: M12, M51, M54, O33

\section{INTRODUCCIÓN}

El trabajo remoto es un factor que ha ido cobrando importancia entre distintos sectores de actividad y empleo desde hace un tiempo, actualmente en el contexto de confinamiento social, preventivo y obligatorio que se vive en el mundo derivado de la pandemia por la COVID-19, se ha vuelto un tema de enorme relevancia (Bonavida, 2020).

La disrupción de las medidas de confinamiento, hicieron que los trabajadores tuvieran que adaptarse a formas de trabajo flexibles y a un nuevo entorno en un período de tiempo muy breve. Para algunos trabajadores, el migrar sus actividades profesionales al trabajo remoto implicó muchas adversidades, se enfrentaron a situaciones diversas, como el acceso a la tecnología, la gestión del tiempo y las tareas, así como la fusión sin precedentes del trabajo y la vida privada y familiar, en muchos casos derivando en efectos en la salud y el bienestar personal de los trabajadores Vol. 7 Núm. 1 Enero-Junio 2021 
En México el trabajo remoto durante la pandemia no pudo ser aplicado de manera generalizada, debido entre otras cosas a que, no se cuenta con las herramientas y la conectividad necesaria para llevarlo a cabo (Camargo, 2020). De acuerdo a la Secretaría del Trabajo y Previsión Social, solamente el $70 \%$ de las actividades laborales pueden desarrollarse de manera remota (González, 2020). Otros datos importantes refieren que, solo dos de cada 10 unidades económicas cuentan con los requerimientos para implementar el trabajo remoto y el $81 \%$ de los empleados no recibe el apoyo tecnológico de la empresa para hacer home office (Ibidem).

El objetivo de presente artículo es analizar las condiciones de acceso a la tecnología para el trabajo remoto en México durante la pandemia de COVID19. Se realiza un análisis comparativo de las condiciones para el trabajo remoto en diferentes sectores económicos, el estudio analiza variables como problemas de acceso a internet, proveedores de servicio, herramientas de trabajo, capacitación, comunicación, problemas digitales, entre otros. El levantamiento de datos se lleva cabo cinco meses después de que los trabajadores trasladaran su actividad laboral a casa, permitiendo esto un período de adaptación a la nueva normalidad. Para realizar el estudio 1685 encuestas a trabajadores de diferentes sectores económicos fueron aplicadas. Los resultados muestran las condiciones de acceso a la tecnología con las que los trabajadores han estado desarrollando el trabajo durante aproximadamente 6 meses de confinamiento.

\section{MARCO TEÓRICO}

\subsection{Impacto económico por la pandemia de la COVID-19}

El 11 de marzo de 2020, la Organización Mundial de la Salud (OMS) calificó el nuevo brote de coronavirus como una pandemia e instó a los gobiernos del mundo a enfrentar este problema con seriedad y a prepararse para la primera ola de la emergencia de salud pública con varias medidas drásticas entre las que se destacan, cierre de escuelas, cierre de lugares de trabajo, restricciones de viaje, cuarentena, distanciamiento social, confinamiento, entre otras (OMS, 2020; OIT, 2020). De acuerdo a datos de la Universidad Johns Hopkins (2021), para el 13 de abril del 2021 se han registrado alrededor de 136.8 millones de casos de coronavirus y 2.9 millones de personas fallecidas en el mundo.

El impacto que las medidas de confinamiento han tendido en la economía de los países ha sido devastador, para el año 2020 algunas predicciones se referían a que, por ejemplo, la economía mundial tendría su mayor caída desde la segunda guerra mundial y de acuerdo al Banco Mundial ésta podría reducirse un 5.2\% (CEPAL, 2020; BM, 2020); habría una disminución del PIB per cápita en el 90\% de los países; la actividad económica de las economías avanzadas se contraería un $7 \%$; los mercados emergentes y las economías en desarrollo se contraerían un $2.5 \%$; la disminución prevista en los ingresos per cápita, de un $3.6 \%$ empujaría a millones de personas a la pobreza extrema (CEPAL, 2020).

En cuanto al impacto en el empleo, de acuerdo a datos de la OIT (2020_b), para 2020 el 93\% de los trabajadores residía en países en los que se aplicó algún tipo de medida de cierre de lugares de trabajo, el $77 \%$ de los trabajadores seguían viéndose afectados por esas medidas a comienzos del año 2021; se perdió el $8.8 \%$ de las horas de trabajo a nivel mundial equivalentes a 255 millones de empleos a tiempo completo; se estima que los ingresos provenientes del trabajo a escala mundial disminuyeron un $8.3 \%$ por ciento correspondiente al $4.4 \%$ del PIB mundial.

El impacto económico por la pandemia en México se ha traducido en la pérdida de empleos y la reducción de ingresos para millones de mexicanos. El Producto Interno Bruto, registró en 2020 una caída de $8.3 \%$, y se perdieron alrededor de 647,710 puestos de trabajo (Rodríguez, et.al., 2021).

\subsection{Trabajo remoto}

El concepto de flexibilidad laboral tiene un auge en la década de los ochenta debido a la necesidad de establecer medidas que lograran mejorar la rigidez que existía en las relaciones laborales, aumentar los niveles de empleo y alcanzar una mejor competitividad (Ibarra \& González, 2010). Para la Organización para la Cooperación y el Desarrollo Económico y el Banco Mundial, la flexibilidad consiste en eliminar o desregular el mercado laboral con el objetivo de acabar con las rigideces causantes de un alto índice de desempleo, así como de un sector informal creciente (Piedrahita, et.al., 2013). Desde un punto de vista social, la flexibilidad puede definirse como la capacidad de los individuos, en la vida económica y en particular en el mercado de trabajo, de renunciar a sus hábitos y 
adaptarse a las nuevas circunstancias (Dahrendorf, 1986; Ibarra \& González, 2010).

Entre las formas de trabajo flexibles se reconocen términos como trabajo remoto (Staples, et.al., 1999; Colbert, et.al., 2016), teletrabajo (Wellman, et.al., 1996; Bailey, 2002), trabajo en casa (Holgersen, et.al., 2021), trabajo móvil (Crawford, et.al., 2011) o e-trabajo (Ramos, et.al., 2020).

El trabajo remoto es una modalidad de trabajo flexible (Ramos, et.al., 2020), el término surgió en Estados Unidos en la década de los ochenta (Selma, 2016). Una de las primeras definiciones asociada es este término se refiere a "cualquier forma de sustitución de desplazamientos relacionados con la actividad laboral por tecnologías de la información" (Niles, 1973; Selma, 2016). La Organización Internacional del Trabajo, define al trabajo remoto con base en dos componentes (OIT, 2020): a) El trabajo se realiza plena o parcialmente en una ubicación alternativa distinta del lugar de trabajo predeterminado y b) La utilización de dispositivos electrónicos personales, como una computadora, una tableta o un teléfono (móvil o fijo) para desempeñar el trabajo. La utilización de dispositivos electrónicos personales debe constituir una parte fundamental del desempeño del trabajo. Osio (2010) define el trabajo remoto a partir de la fórmula: Trabajo remoto $=$ Trabajo + Distancia + Uso intensivo de las TIC.

El trabajo remoto ofrece muchas ventajas tanto para las empresas como para las personas. El trabajo se realiza sin una ubicación u horarios específicos, trabajar en cualquier momento, cualquier lugar y a cualquier hora son algunos de los beneficios que ofrece a las personas (Montaudon, et.al., 2021), permite alcanzar un mejor equilibrio entre la vida laboral, familiar y personal, aumenta la flexibilidad, la productividad, reduce tiempos de desplazamiento. Las organizaciones se hacen más ágiles, flexibles y competitivas además de reducir algunos costos fijos como luz, electricidad, calefacción, espacios físicos, etc., de igual manera abona al medioambiente al disminuir el congestionamiento del tráfico, mejorar las condiciones de las viviendas, evitar hacinamiento en las ciudades, etc., abona también a la igualdad, al facilitar la inserción al mundo laboral de personas con alguna discapacidad, entre otros (Osio, 2010, Buffer, 2019).
Cada vez son más las personas y empresas que optan por esta modalidad de empleo, el trabajo remoto ya no es una tendencia, llegó para quedarse, los beneficios de la deslocalización laboral, el internet y los nuevos perfiles laborales permiten que el realizar teletrabajo de forma efectiva sea posible (Buffer, 2019).

En el año 2020 a raíz de las medidas de confinamiento derivadas de la pandemia de COVID19 el trabajo remoto tuvo un crecimiento muy significativo a nivel global, la pandemia aceleró la adopción de trabajos flexibles porque éstos permitieron a muchas organizaciones la continuidad operativa (OIT, 2020).

De acuerdo a la Organización Internacional del Trabajo (2020), los años venideros posteriores a la pandemia de COVID-19 serán muy inciertos, los trabajadores, empleadores y gobiernos tendrán que adaptarse a una nueva forma de vida y trabajo, que requerirá nuevos comportamientos y nuevas normas, con una gran posibilidad que ello implique una forma híbrida o mixta de aislamiento y de desconfinamiento, por lo que, el trabajo remoto será necesario y fundamental para la operatividad de las organizaciones en el mundo.

En México, el trabajo remoto no es una modalidad de trabajo flexible reciente, hay referencias importantes de su desarrollo, sobre todo por empresas transnacionales radicadas en el país (Jiménez, et.al., 2015). Para el año 2017 de acuerdo al informe Influencia de las Tecnologías de la Información y Comunicación (TIC) sobre la salud de los Trabajadores (Gil-Monte, 2017), México se encontraba entre los países que encabezaban la tendencia de actividad laboral mediante el trabajo remoto, el informe resalta que, para el 2017 el 2.5\% de la población laboral activa en México trabajaba en modalidad remota.

La contingencia sanitaria derivada por la pandemia de COVID-19 en 2020 y las medidas de contingencia asumidas la mayoría de los países del mundo, que incluyeron la suspensión de ciertas actividades económicas, la restricción de congregaciones masivas, la recomendación de resguardo domiciliario a la población general, así como la suspensión de actividades escolares presenciales, entre otras (Secretaría Salud, 2020), detonaron un aumento abrupto y significativo de esta modalidad de trabajo, en México alrededor del 72\% de los trabajadores se encontraba trabajando remotamente posterior a las medidas de confinamiento (STATISTA, 2020). 
En 2020 en México se comenzó a analizar la necesidad de reformar la Ley Federal del Trabajo para regular el trabajo remoto, el 12 de enero del 2021 entra en vigor la reforma al artículo 311 de ley en materia de teletrabajo (DOF, 2021) que establece nueve derechos básicos de las personas que laboran bajo este esquema:

1. Recibir los equipos necesarios para realizar las actividades laborales

2. Recibir su salario/pago en la forma y fechas estipuladas

3. Percibir los costos derivados del teletrabajo como telecomunicación y electricidad

4. Trato correcto de la información y los datos utilizados

5. Desconexión al término de la jornada laboral

6. Mantener sus derechos a la seguridad social

7. Capacitación y asesoría para garantizar la adaptación, aprendizaje y uso adecuado de las tecnologías requeridas.

8. Mantener una relación equilibrada a fin de gozar un trabajo digno o decente

9. Perspectiva de género que permita a las mujeres conciliar la vida familiar y laboral

Establece además como responsabilidad de los empleados:

1. Cuidar de los equipos y materiales que reciban

2. Informar oportunamente sobre los costos derivados del trabajo: servicios de telecomunicación y electricidad.

3. Conducirse con apego a la disposición en materia de seguridad y salud en el trabajo

4. Utilizar los mecanismos y sistemas operativos para la supervisión de sus actividades

5. Atender los mecanismos de protección de datos utilizados en las actividades $\mathrm{y}$ restricciones sobre su uso y almacenamiento.

De manera adicional la Norma Oficial Mexicana NOM-035 (DOF, 2018) emitida por la Secretaría del Trabajo y Previsión Social en 2018, establece las condiciones básicas y necesarias de trabajo en los apartados de seguridad, medio ambiente y salud, con el objetivo de prevenir accidentes laborales y enfermedades provocadas por el trabajo. En este sentido, cuando se analiza el trabajo remoto, se advierte la necesidad de evaluar la recuperación y el descanso de los trabajadores, las cargas laborales excesivas, la extensión de la jornada laboral, la falta de control sobre el trabajo y la interferencia en la relación trabajo-familia, entre otros.
$\mathrm{Si}$ bien el trabajo flexible en condiciones normales conlleva muchos beneficios, la rapidez con que las restricciones y medidas de confinamiento se tuvieron que adoptar así como la forma tan disruptiva de realizar actividades laborales a través del trabajo remoto, derivaron en condiciones de trabajo no idóneas para algunos sectores económicos ya que los trabajadores enfrentaron situaciones muy adversas relacionadas con las condiciones en los hogares, el acceso a la tecnología, la gestión del tiempo y las tareas, el desempeño laboral, las habilidades digitales, así como la fusión sin precedentes del trabajo y la vida privada y familiar (Montaudon, et.al., 2021), en muchos casos derivando en efectos en la salud y el bienestar de los trabajadores.

\subsection{El trabajo remoto y el acceso a la tecnología}

El acceso a las Tecnologías de la Información y la Comunicación (TIC) constituye el requerimiento principal para realizar el trabajo remoto de forma efectiva (Pérez, et.al., 2004; Ramos, et.al., 2020), los trabajadores requieren del conocimiento y uso intensivo de las TIC en el desarrollo de sus actividades (Osio, 2010).

Contar con un servicio de internet confiable, sin interrupciones en la señal es tan importante como los dispositivos que soporten el tipo de trabajo que se realiza, contar con las herramientas digitales y la capacitación para el uso de la tecnología son factores necesarios. En 2020 había en México 80.6 millones de usuarios de internet, sin embargo, existen aún importantes brechas de acceso en algunas zonas urbanas y rurales, solamente el $44.3 \%$ de los hogares mexicanos cuenta con al menos una computadora propia (IFT, 2020).

Debido a las medidas de confinamiento derivadas de la contingencia sanitaria por COVID19, los hogares se volvieron el centro de trabajo, pero también se volvieron el lugar desde donde los hijos atienden las actividades escolares, en México para el ciclo escolar 2020-2021 más de 30 millones de estudiantes y 1.2 millones de docentes, lo iniciaron de forma remota (SEP, 2020) generando un impacto importante en el acceso a la tecnología de cada uno de los hogares.

\subsection{Sectores económicos}

Los sectores económicos son la división de la actividad económica de un estado o territorio y se 
clasifican en primario, secundario, terciario, cuaternario y quinario (Kenessey, 2012). El sector primario agrupa principalmente a sectores que obtienen productos directamente de la naturaleza, materias primas, creaciones, etc., el secundario agrupa sectores que transforman materias primas en productos terminados o semielaborados, el sector terciario es considerado como sector de servicios, en el siglo XXI se subclasifica este sector y se propone el sector cuaternario, este sector agrupa actividades económicas basadas en conocimiento e imposibles de mecanizar, tales como, la generación e intercambio de información, tecnología, consultoría, educación, investigación y desarrollo, planificación financiera, entre otros servicios o actividades principalmente intelectuales (Selstad, 1990; Rosenberg, 2020), el sector quinario incluye servicios sin ánimo de lucro así como actividades domésticas como las realizadas por amas de casa o familiares que cuidan a otros en sus propios hogares (Hatt, et.al., 1953).

En México el sector primario agrupa a sectores como agricultura, ganadería, silvicultura, caza y pesca. El sector secundario agrupa principalmente a la industria extractiva y de la electricidad, industria manufacturera e industria de la construcción, y en el sector terciario se agrupan principalmente, comercio; restaurantes y servicios de alojamiento; transportes, comunicaciones, correo y almacenamiento; servicios profesionales, financieros y corporativos; servicios sociales; servicios diversos; gobierno y organismos internacionales (INEGI, 2021).

\section{MÉTODO}

\subsection{Proceso de investigación}

El levantamiento de datos primarios que fundamentan la presente investigación se llevó a cabo en los meses de agosto a noviembre del año 2020 , entre 5 y 8 meses después de que las medidas de confinamiento y particularmente de resguardo domiciliario fueron declaradas por las autoridades de la Secretaría de Salud en México y acatadas por los 32 estados de la república mexicana. Es importante mencionar que, para el momento en que se recabaron los datos, los trabajadores se encontraban habituados al trabajo remoto al llevar desarrollándolo entre 5 y 8 meses consecutivos.

\subsection{Diseño de la investigación}

La investigación es de tipo cuantitativa con diseño no experimental transversal-descriptivo. Es no experimental con alcance descriptivo (HernándezSampieri, et.al., 2108) ya que tiene como finalidad analizar los efectos del acceso a la tecnología en el trabajo remoto a partir de un conjunto de variables y estimar su ocurrencia; es transversal porque la recolección de datos se realiza en un solo período de tiempo en una muestra representativa de la población (Martínez, et.al., 2011; Hernández-Sampieri, et.al., 2018). La técnica de muestreo es no probabilística a través del diseño de una escala enviada por medios electrónicos.

\subsection{Escala y muestra}

En México, de acuerdo a la Encuesta Nacional de Ocupación y Empleo (ENOE) para febrero del 2021 hay una Población Económicamente Activa (PEA) de aproximadamente 55.5 millones de personas, de las cuales, en el sector primario se concentran 6.7 millones de personas (12\%), en el sector secundario 14.7 millones $(26.5 \%)$ y el sector terciario concentra la mayor parte con 33.9 millones de personas que corresponde al $61 \%$ del total de la PEA (INEGI, 2021).

Las variables analizadas en esta investigación corresponden a un subconjunto de datos de una escala desarrollada para un estudio mucho más amplio que evalúa las condiciones y los efectos del trabajo remoto en diferentes dimensiones de la vida laboral y personal en

trabajadores que derivado de la emergencia sanitaria trasladaron sus actividades profesionales a modalidad teletrabajo.

La escala diseñada está compuesta por varias dimensiones a saber: efectos en el empleo, ingreso y gasto; condiciones en los hogares; acceso a la tecnología y habilidades digitales; gestión del tiempo y las tareas; desempeño laboral; salud y bienestar laboral, relaciones familiares y el futuro del trabajo remoto.

La dimensión acceso a la tecnología y habilidades digitales es la analizada en el presente estudio y se consideran a trabajadores de los diferentes sectores económicos, esta dimensión está compuesta por 30 variables, que incluyen datos de clasificación, preguntas dicotómicas, preguntas de opción múltiple y preguntas en escala de Likert de 7 puntos.

\subsection{Validación}


La muestra para el estudio completo fue levantada en México entre los meses de agosto y noviembre del 2020, es representativa de la población de estudio a nivel nacional, consta de 2,136 observaciones con 113 variables.

La validación de la escala se realiza con el Alpha de Cronbach (Quero, 2010), arrojando un resultado de 0.9235 lo que muestra que es altamente confiable, de acuerdo a George \& Mallery (2003)

Tabla 1. Resultados Alpha de Cronbach

\begin{tabular}{|c|c|c|c|}
\hline Item & Indicador & Código & $\begin{array}{l}\text { Alpha de } \\
\text { Cronbach }\end{array}$ \\
\hline 1 & Edad & edad & 0.9237 \\
\hline 2 & Género & genero & 0.9241 \\
\hline 3 & Estado civil & civil & 0.9243 \\
\hline 4 & Lugar de residencia & estado & 0.9243 \\
\hline 5 & Sector en el que se desempeña & sector & 0.9242 \\
\hline 6 & Número de personas que habitan en su hogar & personas_hog & 0.9238 \\
\hline 7 & $\begin{array}{l}\text { Número de personas en su hogar que trabajan y/o } \\
\text { estudian desde casa durante la pandemia }\end{array}$ & trabajocasa_total & 0.9240 \\
\hline 8 & Compañía proveedora de servicios de internet & internet & 0.9242 \\
\hline 9 & $\begin{array}{l}\text { Número de computadoras o dispositivos digitales que } \\
\text { se utilizan en su hogar para actividades de trabajo o } \\
\text { estudio }\end{array}$ & dispositivo_total & 0.9244 \\
\hline 10 & ¿Tiene computadora propia? & comp_propia & 0.9241 \\
\hline 11 & $\begin{array}{l}\text { Al moverse al trabajo remoto ¿se llevó la computadora } \\
\text { de la oficina a la casa? }\end{array}$ & comp_oficina & 0.9240 \\
\hline 12 & Tiene una conexión a internet confiable & conexión & 0.9228 \\
\hline 13 & $\begin{array}{l}\text { ¿Cuántos miembros de la familia comparten el } \\
\text { internet? }\end{array}$ & internet_comp & 0.9238 \\
\hline 14 & $\begin{array}{l}\text { Experimenta con frecuencia fallas del servicio de } \\
\text { internet }\end{array}$ & fallas & 0.9231 \\
\hline 15 & $\begin{array}{l}\text { ¿Tiene problemas con el internet en horarios } \\
\text { específicos? }\end{array}$ & horario_fallas & 0.9232 \\
\hline 16 & $\begin{array}{l}\text { Utiliza su teléfono celular como herramienta de trabajo } \\
\text { (WhatsApp, etc.) }\end{array}$ & celular & 0.9241 \\
\hline 17 & $\begin{array}{l}\text { ¿Qué software de vídeo conferencia utiliza con mayor } \\
\text { frecuencia? }\end{array}$ & software1 & 0.9243 \\
\hline 18 & Total de softwares de vídeo conferencia que utiliza & total_software & 0.9243 \\
\hline 19 & $\begin{array}{l}\text { Ha recibido la capacitación que requiere para realizar } \\
\text { su trabajo desde casa en forma adecuada }\end{array}$ & capacitación & 0.9235 \\
\hline 20 & $\begin{array}{l}\text { Su empleo le ha facilitado las herramientas de } \\
\text { comunicación necesarias para realizar su trabajo }\end{array}$ & herramientas_com & 0.9230 \\
\hline 21 & $\begin{array}{l}\text { Ha recibido apoyo de los miembros de su familia para } \\
\text { desarrollar su trabajo empleando la tecnología }\end{array}$ & apoyo_tecnología & 0.9243 \\
\hline 22 & $\begin{array}{l}\text { Ha tenido problemas por dejar la cámara o micrófono } \\
\text { abierto sin querer }\end{array}$ & micrófono-cámara & 0.9231 \\
\hline 23 & $\begin{array}{l}\text { Se le ha olvidado activar el micrófono y ha comenzado } \\
\text { a hablar sin que los demás puedan escucharlo }\end{array}$ & no-escucha & 0.9235 \\
\hline 24 & $\begin{array}{l}\text { Antes de la pandemia consideraba que tenía las } \\
\text { habilidades digitales necesarias para llevar a cabo su } \\
\text { trabajo desde casa }\end{array}$ & habilidad_previa & 0.9230 \\
\hline 25 & $\begin{array}{l}\text { Ha tenido dificultades al utilizar ciertas herramientas } \\
\text { digitales para su trabajo }\end{array}$ & dificultad_digital & 0.9225 \\
\hline 26 & $\begin{array}{l}\text { Considera que desde el inicio de la pandemia ha } \\
\text { desarrollado las habilidades digitales necesarias }\end{array}$ & desarrollo_habilidad & 0.9237 \\
\hline
\end{tabular}

los valores arriba de 0.9 tienen una ponderación de excelente. Para este análisis se consideran 1685 observaciones y 28 variables. El Alpha de Cronbach de cada una de las variables estudiadas en este artículo se presenta en la Tabla 1. El software utilizado para realizar el cálculo es STATA. 


\begin{tabular}{|l|l|l|l|}
\hline 27 & $\begin{array}{l}\text { Ha sido difícil adaptarse a las herramientas digitales } \\
\text { proporcionadas por la empresa }\end{array}$ & adaptación & 0.9222 \\
\hline 28 & $\begin{array}{l}\text { El tener que utilizar diferentes tecnologías ha sido } \\
\text { difícil }\end{array}$ & dificultad & 0.9217 \\
\hline
\end{tabular}

Fuente: Elaboración propia

\section{RESULTADOS Y ANÁLISIS}

Los datos de clasificación muestran que el promedio de edad de los participantes es de 46 años en un rango de 18 a 79 años. El 54.9\% corresponde al género femenino, el $44.8 \%$ al género masculino y el $0.3 \%$ prefiere no especificar. En cuanto al estado civil, el $53.88 \%$ es casada/o, $33.14 \%$ soltera/o, $1.13 \%$ viuda/o, $7.17 \%$ divorciada/o y
4.68\% unión libre.

Los participantes se encuentran ubicados en 30 de los 32 estados de la república mexicana, el porcentaje de participación en cada estado se muestra en la Figura 1.

Figura 1. Distribución de la muestra por estado

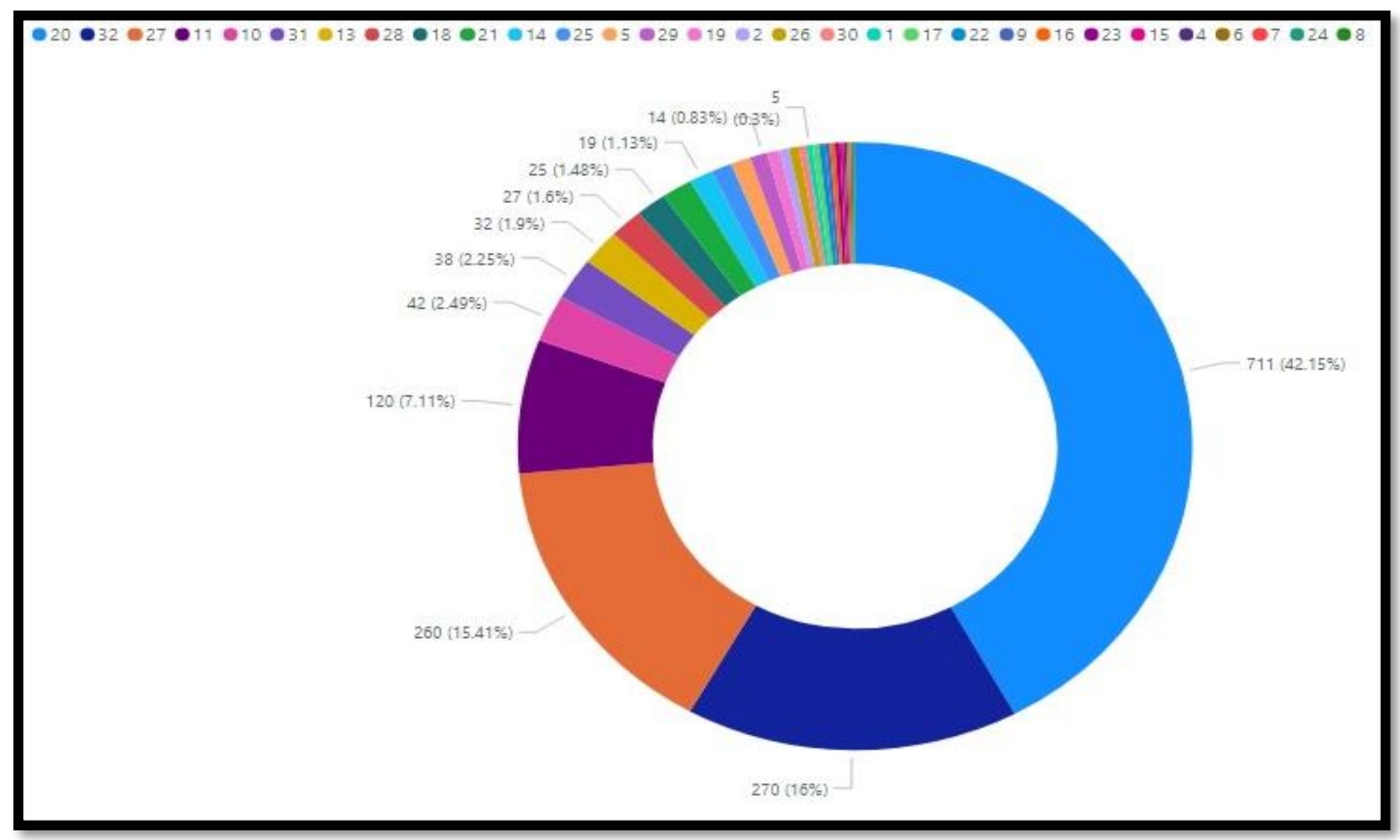

Fuente: Elaboración propia. Abreviaturas: 1. Aguascalientes, 2. Baja California, 4. Campeche, 5. Chiapas, 6. Chihuahua. 7. Coahuila de Zaragoza, 8. Colima, 9. Durango, 10. Estado de México, 11. Estado de México, 13. Hidalgo, 14. Jalisco, 15. Michoacán de Ocampo, 16. Morelos, 17. Nayarit, 18. Nuevo León, 19. Oaxaca, 20. Puebla, 21. Querétaro, 22. Quintana Roo, 23. San Luis Potosí, 24. Sinaloa, 25. Sonora, 26. Tabasco, 27. Tamaulipas, 28. Tlaxcala, 28, Veracruz de la Llave, 30. Yucatán, 31. Zacatecas, 32. Ciudad de México CDMX.

Los participantes se encuentran distribuidos en diferentes sectores económicos, al sector primario corresponde el $0.89 \%$ de la población estudiada, al sector secundario el $6.99 \%$, al sector terciario el $28.75 \%$, al sector cuaternario el $61.76 \%$ y al sector quinario el $1.36 \%$ de la población estudiada. El $0.2 \%$ de la población no contestó a esta pregunta. El número de participantes por cada sector económico se muestra en la Figura 2.

Figura 2. Participantes por sector económico 


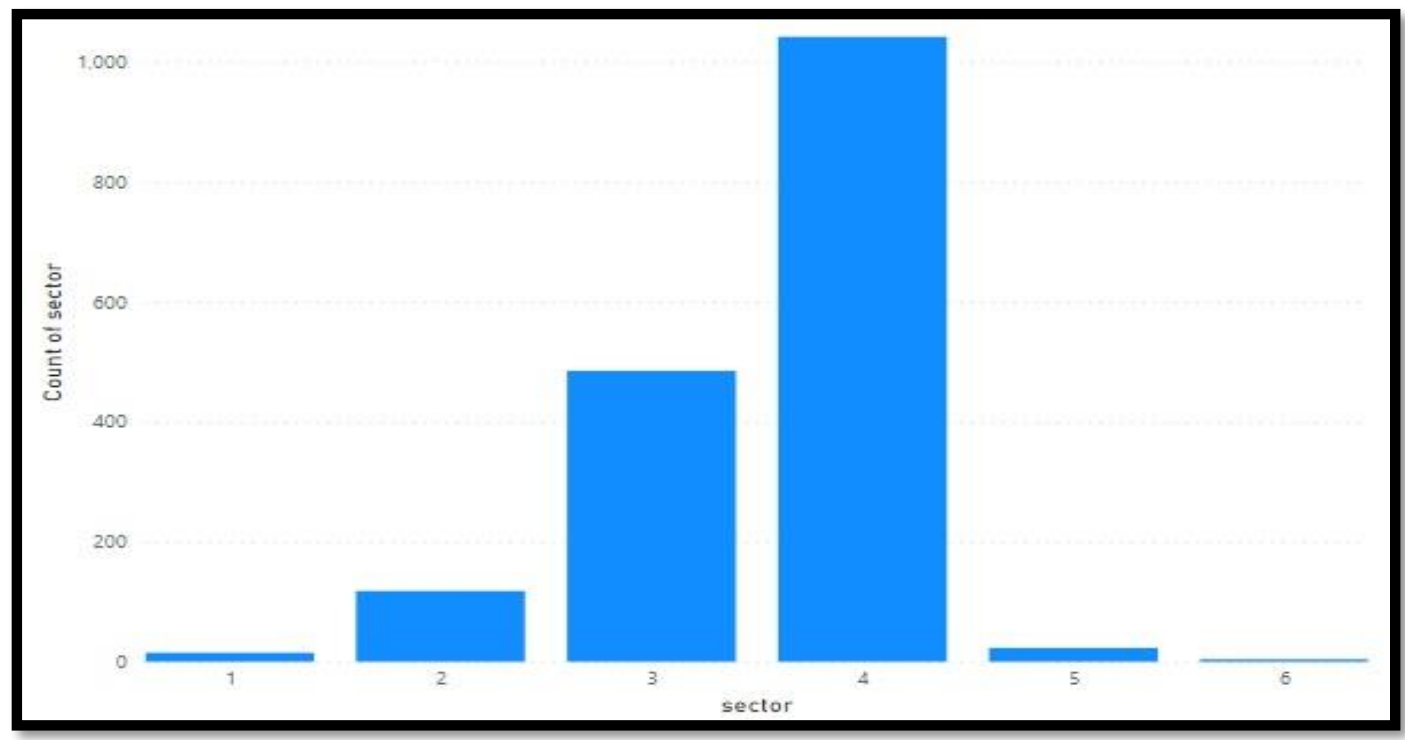

Fuente: Elaboración propia. Abreviaturas: 1. Sector primario, 2. Sector secundario, 3. Sector terciario, 4. Sector cuaternario, 5. Sector quinario, 6. No contestaron

En promedio 2.5 personas trabajan y/o estudian en el hogar durante el confinamiento, el promedio de dispositivos en el hogar es de 3.41 considerando el uso de diferentes dispositivos que incluyen computadoras de escritorio, laptops, tabletas y teléfonos celulares (Ver Tabla 2). El 88.8\% utiliza el teléfono celular como herramienta de trabajo.

Tabla 2. Número de dispositivos VS Número de personas que trabajan y/o estudian

\begin{tabular}{|c|c|c|c|c|c|c|}
\hline$\#$ & \multicolumn{6}{|c|}{ No. de personas que trabajan y/o estudian en casa (\%) } \\
\cline { 2 - 7 } Dispositivos & 1 & 2 & 3 & 4 & 5 & $>=6$ \\
\hline 1 & 4.9 & 1.8 & 0.6 & 0.5 & 0.06 & 0 \\
\hline 2 & 8.8 & 10.3 & 1.8 & 0.8 & 0.3 & 0.1 \\
\hline 3 & 4.7 & 7.0 & 9.5 & 1.5 & 0.4 & 0.2 \\
\hline 4 & 1.5 & 6.3 & 5.1 & 7.4 & 0.9 & 0.2 \\
\hline 5 & 0.5 & 2.0 & 2.6 & 1.1 & 1.7 & 0.1 \\
\hline$>=6$ & 1.0 & 1.9 & 4.7 & 4.5 & 1.9 & 0.7 \\
\hline
\end{tabular}

Fuente: Elaboración propia. El 2.6\% de la población estudiada no respondió a alguna de las dos preguntas o a ambas.

Como se observa en la Tabla 2, si se considera solamente el número de personas que utilizan algún dispositivo para trabajar y/o estudiar, aunque la mayoría de los entrevistados cubre sus necesidades, algunos otros no. Se destaca que, de los que cuentan con un dispositivo el $2.96 \%$ requieren dos o más; de los que cuentan con dos dispositivos, el 3\% requieren tres o más; de los que cuentan con tres dispositivos, el $2.1 \%$ requieren cuatro o más; de los que cuentan con cuatro dispositivos, el $1.1 \%$ requieren cinco o más y de los que cuentan con cinco dispositivos, el $0.1 \%$ requieren seis o más.
Si se considera el número de personas en la familia que comparten el internet incluyendo no solamente a las personas que trabajan y/o estudian sino a otros miembros de la familia como abuelos o hermanos menores, las necesidades de dispositivos aumentan (ver Tabla 3). En promedio 3.5 personas por familia comparten el internet. El $78.69 \%$ necesita entre 2 y 4 dispositivos para cubrir sus necesidades. El 68\% de los encuestados reporta tener entre 2 y 4 dispositivos en casa, por lo que hay una brecha negativa de aproximadamente el $10.69 \%$. 


\begin{tabular}{|c|c|c|c|c|}
\hline $\begin{array}{l}\text { No. de personas en la } \\
\text { familia que comparten el } \\
\text { internet }\end{array}$ & $\begin{array}{c}\text { Porcentaje } \\
(\%)\end{array}$ & $\begin{array}{c}\text { No. de dispositivos que } \\
\text { utiliza en su hogar }\end{array}$ & $\begin{array}{c}\text { Porcentaje } \\
(\%)\end{array}$ & Brecha \\
\hline 1 & 6.82 & 1 & 8.90 & + \\
\hline 2 & 24.51 & 2 & 22.73 & - \\
\hline 3 & 27.06 & 3 & 23.68 & - \\
\hline 4 & 27.12 & 4 & 21.60 & - \\
\hline 5 & 10.26 & 5 & 8.01 & - \\
\hline 6 o más & 4.21 & 6 o más & 14.90 & + \\
\hline
\end{tabular}

En México existen diversas compañías que ofrecen el servicio de internet. En México, el $50.03 \%$ de la población encuestada utiliza Telmex, el $15.83 \%$
Megacable, el $13.04 \%$ Izzi, el 12\% TotalPlay y el 9.13\% están con otras compañías (ver Figura 3 ).

Figura 3. Distribución de compañías de internet

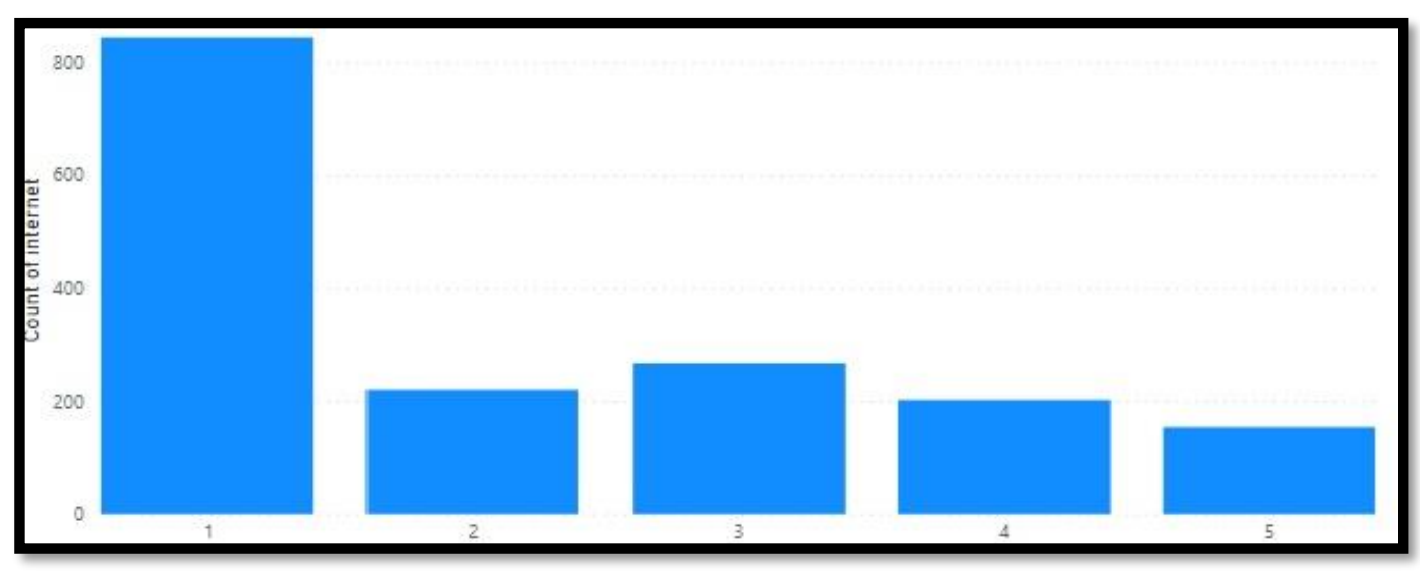

Elaboración propia. Abreviaturas: 1. Telmex, 2. Izzi, 3. Megacable, 4. TotalPlay, 5. Otras compañías

De los 1685 entrevistados, el 39.7\% utiliza Zoom como plataforma de trabajo principalmente para videoconferencias, seguido de Teams con el $38.34 \%$, Meet con el $15.34 \%$, Blackboard con el $3.66 \%$ y el $2.96 \%$ utilizan otras plataformas (ver Figura 4).
En cuanto al número total de softwares que utilizan, el $16.95 \%$ utiliza uno, el $32.73 \%$ utiliza 2 , el $36.39 \%$ utiliza 3 , el $11.61 \%$ utiliza 4 y el $2.32 \%$ utiliza 5 o más softwares. El promedio de softwares utilizados es de 2.04 .

Figura 4. Distribución de plataformas para videoconferencias

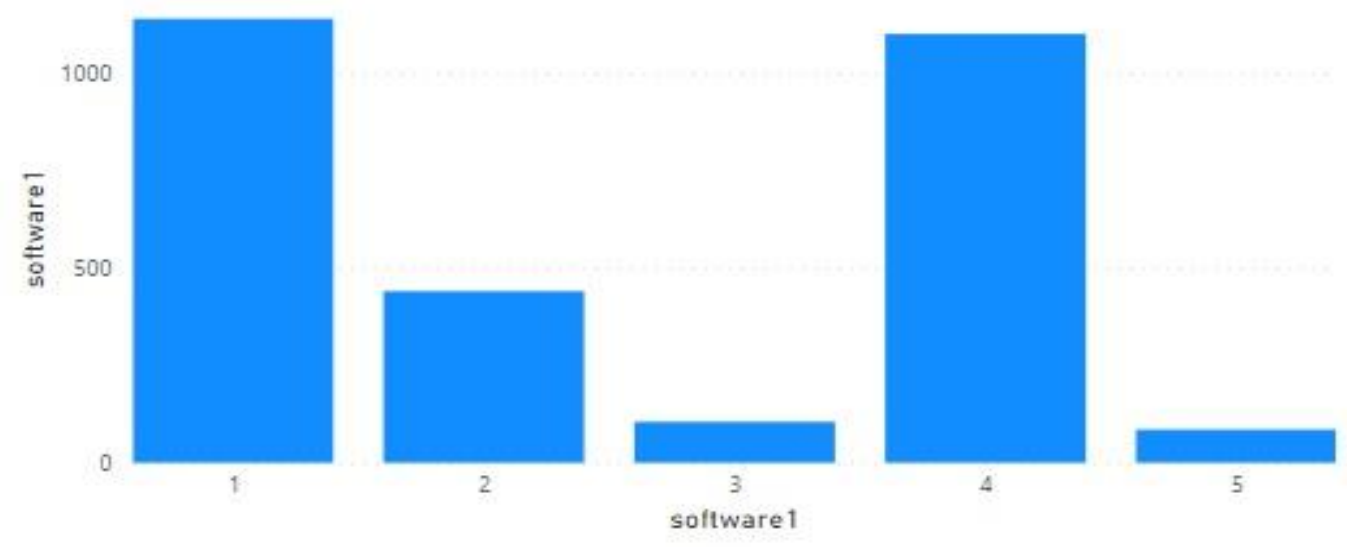

Elaboración propia. Abreviaturas: 1: Zoom, 2. Meet, 3. Blackboard, 4. Teams, 5. Otros

Los resultados de los indicadores formulados en

escala de Likert, se presentan en la Tabla 5. Se 
utiliza una escala de Likert de 7 valores asignados (ver Tabla 4). Se incluye además por cada ítem, la media, desviación estándar y nivel de acuerdo o desacuerdo. Para el nivel de desacuerdo se agrupan los valores del 1 al 3 y para el nivel de acuerdo se agrupan los valores del 5 al 7, el valor 4 se considera neutro. La Tabla 6 agrupa los indicadores formulados como preguntas dicotómicas.

Tabla 4. Valores de la escala de Likert

\begin{tabular}{|c|c|}
\hline Valor & Interpretación \\
\hline 7 & Totalmente de acuerdo \\
\hline 6 & De acuerdo \\
\hline 5 & Parcialmente de acuerdo \\
\hline 4 & Ni de acuerdo ni en desacuerdo \\
\hline 3 & Parcialmente en desacuerdo \\
\hline 2 & En desacuerdo \\
\hline 1 & Totalmente en desacuerdo \\
\hline
\end{tabular}

Tabla 5. Resultados por cada indicador y por sector económico

\begin{tabular}{|c|c|c|c|c|c|c|}
\hline \multirow[t]{2}{*}{ Item } & \multirow[t]{2}{*}{ Indicador } & \multirow[t]{2}{*}{ Media } & \multirow[t]{2}{*}{$\begin{array}{l}\text { Desviación } \\
\text { Stándard }\end{array}$} & \multicolumn{3}{|c|}{$\begin{array}{l}\text { Nivel de acuerdo o desacuerdo } \\
(\%)\end{array}$} \\
\hline & & & & $\begin{array}{l}\text { Desacuerdo } \\
\quad(1-3)\end{array}$ & $\begin{array}{c}\text { Neutral } \\
(4)\end{array}$ & $\begin{array}{c}\text { Acuerdo } \\
(5-7)\end{array}$ \\
\hline \multicolumn{7}{|c|}{ TECNOLOGÍA / CONECTIVIDAD } \\
\hline \multirow[t]{6}{*}{12} & \multicolumn{6}{|c|}{ Tiene una conexión a internet confiable } \\
\hline & Sector primario & 5.27 & 1.12 & 13.33 & 6.66 & 80.00 \\
\hline & Sector secundario & 5.31 & 1.27 & 5.93 & 11.86 & 82.20 \\
\hline & Sector terciario & 5.53 & 1.42 & 9.69 & 11.95 & 78.35 \\
\hline & Sector cuaternario & 5.23 & 1.59 & 14.77 & 13.34 & 71.88 \\
\hline & Sector quinario & 5.39 & 1.41 & 8.69 & 17.39 & 73.91 \\
\hline \multirow[t]{6}{*}{14} & \multicolumn{6}{|c|}{ Experimenta con frecuencia fallas del servicio de internet } \\
\hline & Sector primario & 4.40 & 1.70 & 33.33 & 20.00 & 46.66 \\
\hline & Sector secundario & 4.30 & 1.88 & 38.13 & 13.55 & 43.30 \\
\hline & Sector terciario & 4.14 & 1.95 & 37.73 & 13.81 & 48.45 \\
\hline & Sector cuaternario & 4.07 & 1.94 & 40.78 & 12.09 & 47.12 \\
\hline & Sector quinario & 5.30 & 1.73 & 21.73 & 8.69 & 69.56 \\
\hline \multirow[t]{6}{*}{16} & \multicolumn{6}{|c|}{ Utiliza su teléfono celular como herramienta de trabajo (WhatsApp, etc.) } \\
\hline & Sector primario & 6.73 & 1.00 & 6.66 & 0 & 93.33 \\
\hline & Sector secundario & 6.06 & 1.69 & 11.01 & 5.93 & 83.05 \\
\hline & Sector terciario & 6.53 & 1.20 & 4.12 & 2.68 & 93.19 \\
\hline & Sector cuaternario & 6.22 & 1.53 & 7.58 & 4.51 & 87.90 \\
\hline & Sector quinario & 5.43 & 2.26 & 17.39 & 13.04 & 69.56 \\
\hline \multicolumn{7}{|c|}{ HERRAMIENTAS PARA EL TELETRABAJO } \\
\hline \multirow[t]{6}{*}{20} & \multicolumn{6}{|c|}{$\begin{array}{l}\text { Su empleo le ha facilitado las herramientas de comunicación necesarias para } \\
\text { realizar su trabajo }\end{array}$} \\
\hline & Sector primario & 5.93 & 1.61 & 13.33 & 6.66 & 80.00 \\
\hline & Sector secundario & 5.52 & 1.94 & 16.10 & 11.86 & 72.03 \\
\hline & Sector terciario & 5.25 & 1.93 & 17.31 & 16.49 & 66.18 \\
\hline & Sector cuaternario & 5.06 & 2.07 & 21.40 & 11.70 & 66.89 \\
\hline & Sector quinario & 5.35 & 1.92 & 17.39 & 17.39 & 65.21 \\
\hline \multirow[t]{6}{*}{25} & \multicolumn{6}{|c|}{ Ha tenido dificultades al utilizar ciertas herramientas digitales para su trabajo } \\
\hline & Sector primario & 2.93 & 1.84 & 73.33 & 0 & 26.66 \\
\hline & Sector secundario & 2.88 & 1.94 & 68.64 & 5.93 & 25.42 \\
\hline & Sector terciario & 3.34 & 2.03 & 56.49 & 8.66 & 34.84 \\
\hline & Sector cuaternario & 3.39 & 2.03 & 53.55 & 12.47 & 33.97 \\
\hline & Sector quinario & 3.09 & 2.36 & 60.86 & 13.04 & 30.43 \\
\hline \multirow[t]{3}{*}{26} & \multicolumn{6}{|c|}{$\begin{array}{l}\text { Considera que desde el inicio de la pandemia ha desarrollado las habilidades } \\
\text { digitales necesarias }\end{array}$} \\
\hline & Sector primario & 6.00 & 1.37 & 6.66 & 6.66 & 86.66 \\
\hline & Sector secundario & 5.73 & 1.72 & 10.16 & 11.86 & 77.96 \\
\hline
\end{tabular}




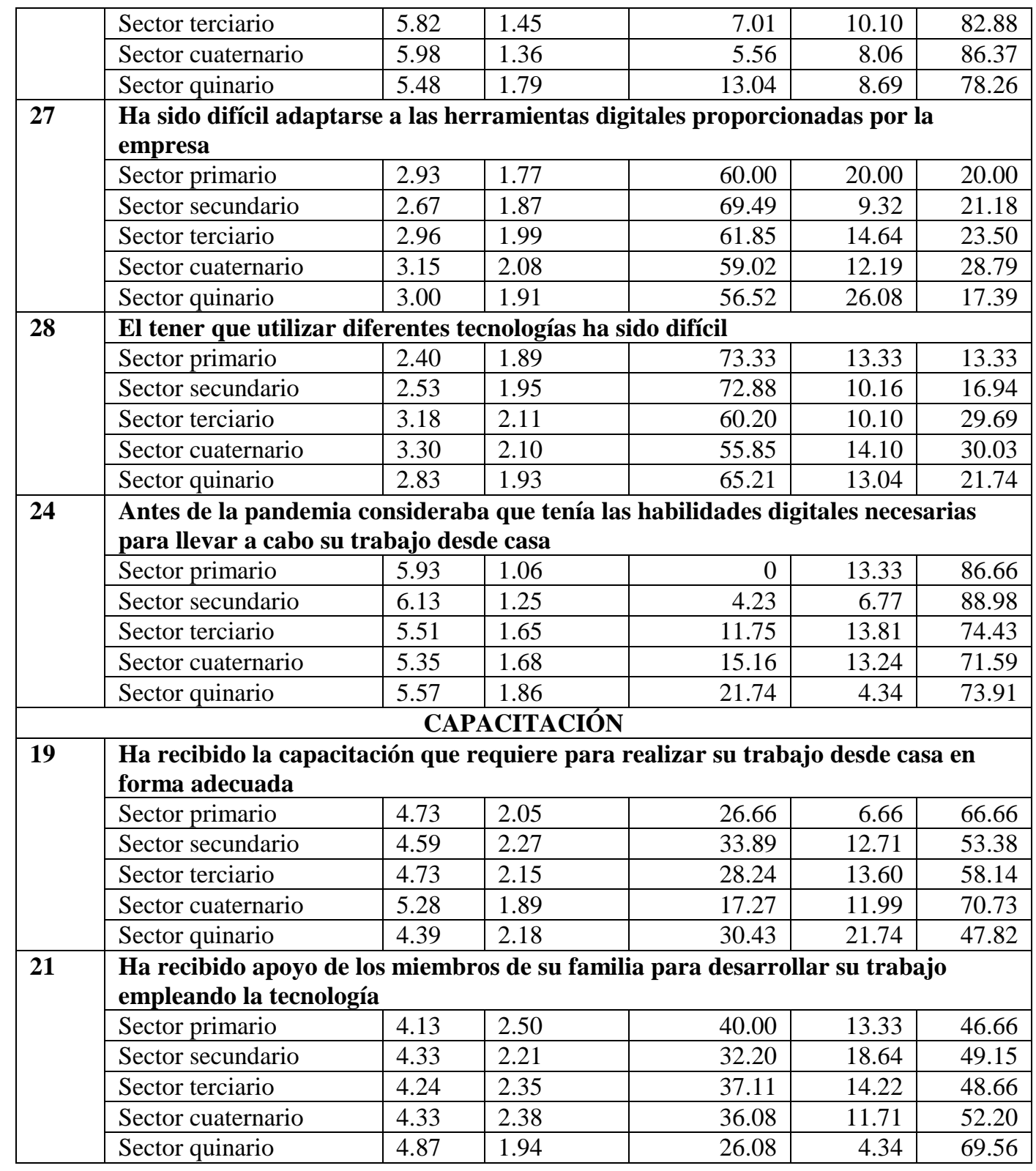

Tabla 6. Resultados preguntas dicotómicas

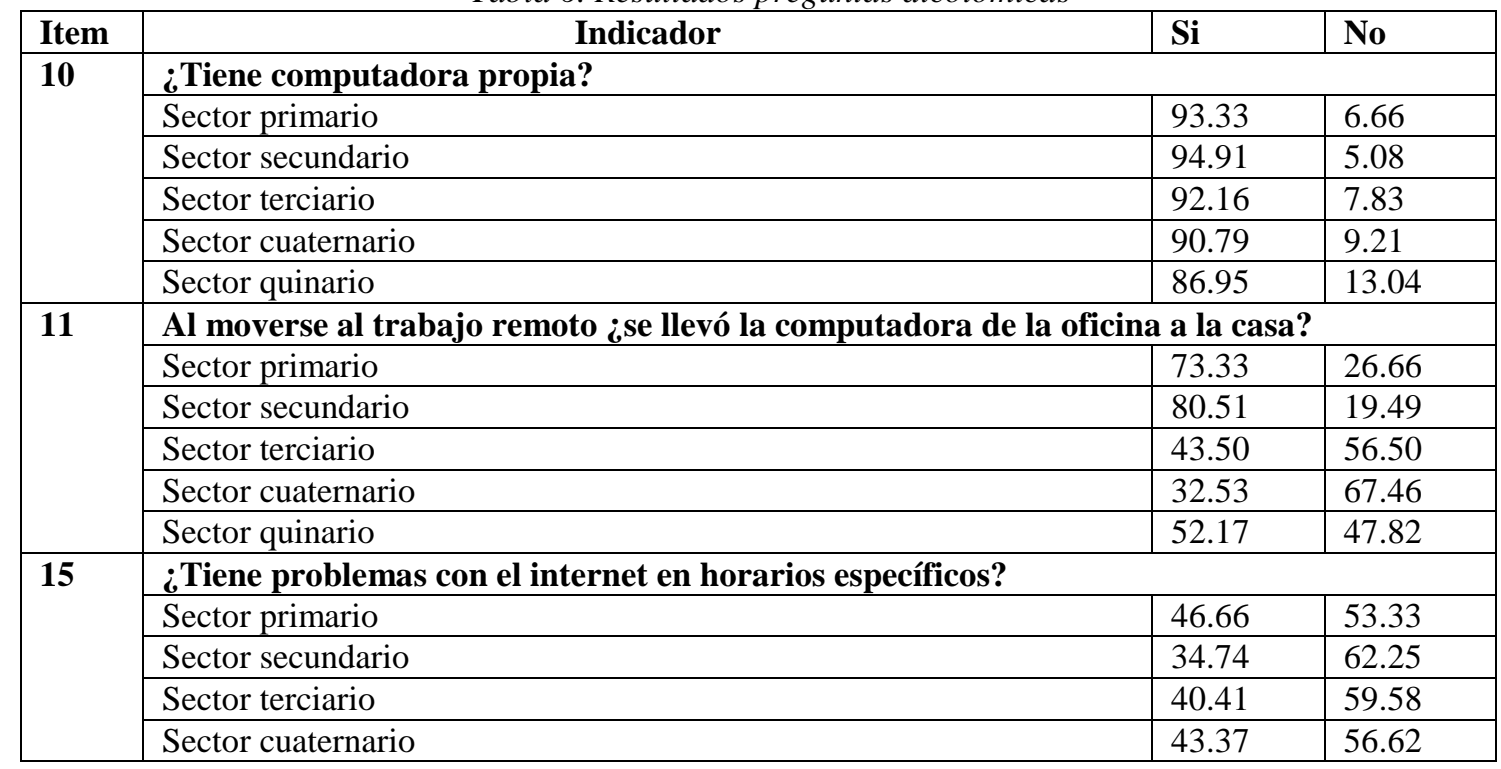




\begin{tabular}{|l|l|l|l|}
\hline \multirow{2}{*}{22} & Sector quinario & 43.47 & 56.52 \\
\cline { 2 - 4 } & Ha tenido problemas por dejar la cámara o micrófono abierto sin querer \\
\cline { 2 - 4 } & Sector primario & 6.66 & 93.33 \\
\cline { 2 - 4 } & Sector secundario & 3.39 & 88.13 \\
\cline { 2 - 4 } & Sector terciario & 13.61 & 86.39 \\
\cline { 2 - 4 } & Sector cuaternario & 13.33 & 86.66 \\
\cline { 2 - 5 } & Sector quinario & 17.39 & 82.61 \\
\hline \multirow{2}{*}{} & $\begin{array}{l}\text { Se le ha olvidado activar el micrófono y ha comenzado a hablar sin que los demás } \\
\text { puedan escucharlo }\end{array}$ & 66.66 & 33.33 \\
\hline & Sector primario & 56.78 & 43.22 \\
\hline & Sector secundario & 53.81 & 46.18 \\
\hline & Sector terciario & 60.55 & 39.44 \\
\hline & Sector cuaternario & 86.95 & 13.04 \\
\hline & Sector quinario & & \multicolumn{2}{|c|}{} \\
\hline
\end{tabular}

\section{CONCLUSIONES}

\section{a) Tecnología / Conectividad}

De acuerdo a los resultados de la Tabla 6 para los diferentes sectores económicos en México, con relación a la tecnología y la conectividad, se destacan las siguientes conclusiones:

$\checkmark$ De acuerdo a los valores de la media que van de 5.23 a 5.53 en una escala de 7 puntos, los participantes del estudio en todos los sectores consideran tener una conexión a internet confiable, los sectores en donde se reportan más problemas con la señal son el sector cuaternario y el sector quinario.

$\checkmark$ Se observa que aun cuando la mayoría considera tener un internet confiable, alrededor de la mitad de la población encuestada manifiesta que experimentan fallas en el servicio, esto se presenta con mayor frecuencia en el sector quinario y terciario, alrededor de la mitad de la población encuestada manifiesta tener problemas con el internet en horarios específicos.

$\checkmark$ Alrededor de 9 de cada 10 personas encuestadas en todos los sectores, manifiestan tener computadora propia, sin embargo, en muchos casos necesitaron llevarse la computadora del trabajo a sus hogares para poder llevar a cabo el trabajo remoto, esto se manifiesta en mayor medida en el sector cuaternario, donde alrededor de 7 de cada 10 trabajadores necesitaron llevarse la computadora del trabajo a su hogar; de igual manera en el sector terciario, alrededor de 6 de cada 10 trabajadores requirieron llevarse la computadora del trabajo a su hogar. Un dato importante se refiere a que, principalmente en el sector primario, secundario, terciario y cuaternario, alrededor de 9 de cada 10 personas utiliza el teléfono celular como herramienta de trabajo.

$\checkmark$ La mayoría de las personas encuestadas manifiesta no haber tenido problemas por dejar la cámara o micrófonos abiertos sin querer, aunque el olvidar activar el micrófono en algún momento es algo más recurrente.

\section{b) Herramientas para el teletrabajo}

Con respecto a las herramientas para el trabajo remoto, se puede concluir que:

$\checkmark \quad$ En su mayoría y en todos los sectores las personas encuestadas manifiestan que, su empresa les facilitó las herramientas de comunicación necesarias para el desarrollo de su trabajo, sin embargo, aún hay una brecha que se debe atender, principalmente en el sector terciario, cuaternario y quinario.

$\checkmark$ Las personas que manifiestan haber tenido más dificultades para utilizar las herramientas digitales que requieren para realizar su trabajo pertenecen a los sectores, terciario, cuaternario $\mathrm{y}$ quinario.

$\checkmark$ Alrededor de 8 de cada 10 personas manifiesta haber consolidado las habilidades digitales necesarias para el desarrollo de su trabajo, aunque, alrededor de 7 de cada 10 considera que éstas habilidades las tenía antes de la pandemia. En este sentido, hay áreas de oportunidad que se requieren atender, principalmente en el sector terciario y cuaternario, ya que son principalmente estos sectores los que manifiestan en 
mayor medida dificultad para adaptarse

a las herramientas digitales proporcionadas por la empresa.

\section{c) Capacitación}

En relación a la capacitación, se puede concluir que:

$\checkmark$ Alrededor de la mitad de los encuestados manifiestan haber recibido la capacitación que requieren para realizar su trabajo desde casa en forma adecuada, sin embargo, hay una brecha digital que se debe atender, principalmente en los sectores secundario y quinario.

$\checkmark$ El apoyo de otros miembros de la familia para el uso de la tecnología y el desarrollo eficiente del trabajo ha sido efectivo en alrededor de la mitad de las personas encuestadas en todos los sectores, principalmente en el quinario y el cuaternario. 


\section{REFERENCIAS}

Bailey, D.E., Kurland, N.B. (2002). A review of telework research: findings, new directions, and lessons for the study of modern work. Journal of Organizational Behavior, 23, 383-400.

Banco Mundial BM. (2020). La COVID-19 (coronavirus) hunde a la economía mundial en la peor recesión desde la Segunda Guerra Mundial. https://www.bancomundial.org/es/news/pressrelease/2020/06/08/covid-19-to-plunge-global-economy-into-worst-recession-since-world-war-ii

Bonavida, C., Gasparini, L. (2020). El Impacto Asimétrico de la Cuarentena. Estimaciones en base a una caracterización de ocupaciones. Documentos de Trabajo del CEDLAS, 261, 1-20.

CIRT. (2020).

Buffer. (2019). State of Remote Work. https://buffer.com/state-of-remote-work-2019

Colbert, A., Yee, N., George, G. (2016). The digital workforce and the workplace of the future. Academy of Management Journal, 59(3), 731-739.

Comisión Económica para América Latina y el Caribe CEPAL. (2020). El impacto económico del COVID-19 y el panorama social hacia 2030 en la región.

Crawford, J.O., MacCalman, L., Jackson, C.A. (2011). The health and well-being of remote and mobile workers. Occupational Medicine, 61(6), 385-394.

Dahrendorf, R. (1986). La flexibilidad del mercado de trabajo. Colección de informes de la OCDE. MTSS Madrid.

Diario Oficial de la Federación DOF (2021). Ley Federal del Trabajo. https://www.gob.mx/cms/uploads/attachment/file/156203/1044_Ley_Federal_del_Trabajo.pdf

Diario Oficial de la Federación DOF (2018). Norma Oficial Mexicana NOM-035-STPS-2018. https://www.dof.gob.mx/nota_detalle.php?codigo $=5541828 \&$ fecha $=23 / 10 / 2018$

Gil-Monte, P.R. (2017). Influencia de las Tecnologías de la Información y de la Comunicación (TIC) sobre la salud de los trabajadores. Reporte Técnico. Universidad Internacional de Valencia

González, E.J. (2020). ¿Home Office en México? Es más fácil decirlo. MILENIO. https://www.milenio.com/opinion/eduardo-javier-gonzalez/punto-porcentual/home-office-en-mexicoes-mas-facil-decirlo

Hatt, P., Foote, N. (1953). On the expansion of the tertiary, quaternary, and quinary sectors. American Economy Review.

Hernández-Sampieri, R., Mendoza, C.P. (2018). Metodología de la Investigación: Las rutas cuantitativa, cualitativa y mixta. Mc Graw Hill

Holgersen, H., Jia, Z., Svenkerud, S. (2021). Who and how many can work from home? Evidence from task descriptions. Journal for Labour Market Research, 55(1), https://doi.org/10.1186/s12651-021-00287-z

Ibarra, M.A., González, L.A. (2010). La flexibilidad laboral como estrategia de competitividad y sus efectos sobre la economía, la empresa y el mercado del trabajo. Contaduría y Administración, 231, 33-52.

Instituto Nacional de Estadística y Geografía INEGI (2021). Resultados de la Encuesta Nacional de Ocupación y Empleo. Cifras Oportunas de Febrero de 2021. https://www.inegi.org.mx/contenidos/saladeprensa/boletines/2021/iooe/iooe2021_03.pdf

Jiménez, A.E., Pérez, R., Martínez, R. (2015). El teletrabajo en México, aspectos normativos y desarrollo. ELAC. Editorial Universitaria.

John Hopkins University (2021). Coronavirus Resource Center. Recuperado de: https://coronavirus.jhu.edu/map.html

Kenessey, Z. (1987). The primary, secondary, tertiary and quaternary sectors of the economy. https://doi.org/10.1111/j.1475-4991.1987.tb00680.x

Martínez, K.I., Ávila, O.P., Pacheco, A., Lira, J. (2011). Investigaciones longitudinales: Su importancia en el estudio del mantenimiento de cambio en las adicciones. Enseñanza e Investigación en Psicología, 16(2), 375-386.

Montaudon-Tomas, C.M., Pinto-López, I.N., Yáñez-Moneda, A.L., Amsler, A. (2021). The Effects of Remote Work on Family Relationships. Chapter in Future of Work, Work-Family Satisfaction, and Employee Well-Being in the Fourth Industrial Revolution. IGI Global.

Niles, J. (1973). The telecommunications-transportation tradeoff. Options for tomorrow and today. California, Jala Internacional.

Osio, L. (2010). El teletrabajo: Una opción en la era digital. Observatorio Laboral Revista Venezolana, 3(5), 93-109.

Organización Internacional del Trabajo OIT. (2020). El teletrabajo durante la pandemia de COVID-19 y

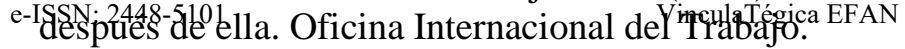


Organización Internacional del Trabajo OIT (2020_b). COVID19: Orientaciones para la recolección de estadísticas del trabajo. Recuperado de: https://www.ilo.org/global/statistics-anddatabases/publications/WCMS 758333/lang--es/index.htm

Organización Mundial de la Salud OMS. (2020). Alocución de apertura del Director General de la OMS en la rueda de prensa sobre la COVID-19. https://www.who.int/es/dg/speeches/detail/who-director-generals-opening-remarks-at-the-media-briefing-on-covid-19---11-march-2020

Pérez, M., Martínez, A., de Luis, P., Vela, M.J. (2004). La adopción del teletrabajo y las tecnologías de la información. Revista de Economía y Empresa, 52-53, 11-27.

Piedrahita, D.A., Angulo, H.A., López, H.E. (2013). Flexibilidad laboral, análisis conceptual y su incidencia en los países latinoamericanos, recientes debates. Prospectiva, 18, 221-242.

Quero, M. (2010). Confiabilidad y coeficiente Alpha de Cronbach. Telos, 12(2), 248-252.

Ramos, V., Ramos-Galarza, C., Tejera, E. (2020). Teletrabajo en tiempos de COVID-19. Interamerican Journal of Psychology, 54(3), 1-30.

Rodríguez, S., Valadez, R., Guzmán, K. (2021). A un año del primer caso de covid en México: impacto económico de pandemia en gráficas. Milenio. https://www.milenio.com/negocios/impacto-economicocovid-19-mexico-7-graficas

Rosenberg, M. (2020). The 5 Sectors of the Economy. Recuperado de: https://www.thoughtco.com/sectors-ofthe-economy-1435795

Secretaría de Educación Pública. (2020). https://www.gob.mx/sep

Secretaría de Salud (2020). Inicia Fase 2 por coronavirus COVID-19. Recuperado de: https://www.gob.mx/salud/prensa/095-inicia-fase-2-por-coronavirus-covid-19

Selstad, T. (1990). The rise of the quaternary sector. The regional dimension of knowledge-based services in Norway, 1970-1985. Norwegian Journal of Geography, 44(1), 21-37. DOI: 10.1080/00291959008552242

Selma, J. (2016). El Teletrabajo, ¿una solución? Facultad de Ciencias Sociales y Jurídicas de Elche.

Staples, D.S., Hulland, J.S., Higgins, C.A. (1999). A Self-Efficacy Theory Explanation for the Management of Remote Workers in Virtual Organizations. Organizations Science, 10(6), 758-776.

STATISTA (2020). Porcentaje de empleados que trabajaron en casa durante el confinamiento en México en junio de 2020. Recuperado de: https://es.statista.com/estadisticas/1147384/porcentaje-empleadoshome-office-mexico/

Wellman, B., Salaff, J., Dimitrova, D., Garton, L., Gulia, M., Haythornthwaite, C. (1996). Computer Network and Social Networks: Collaborative work, Telework, and Virtual Community. Annual Review of Sociology, 22, 213-238. 